\title{
See-Saw Mechanism and Dimensional Reduction in Connection with Exotic Metrics
}

\section{Moises Rojas*}

Universidade Federal de Lavras

E-mail: mrojas@cbpf.br

\section{P. G. De Assis}

Universidade Federal Rural do Rio de Janeiro and Centro Brasileiro de Pesquisas Físicas

E-mail: lpgassis@cbpf.br

\section{José. A. Helayel-Neto}

Centro Brasileiro de Pesquisas Físicas and Grupo de Física Teórica José Leite Lopes

E-mail: helayelecbpf.br

\section{A. De Andrade}

Universidade Estadual do Rio de Janeiro (Resende-RJ)

E-mail: deandrade.marco@gmail.com

\begin{abstract}
We show that Majorana-Weyl spinors of a given chirality in dimensions higher than four can be interpreted, in (1+3)-dimensions, as four Majorana particles with different masses in general. This mechanism borrows a peculiar connection with the See-Saw scheme associated to neutrinos with Majorana-type masses.
\end{abstract}

4th International Conference on Fundamental Interactions -ICFI2010,

August 1-7, 2010

Viçosa, Brazil

\footnotetext{
*Speaker.

${ }^{\dagger}$ the author has moved to $\mathrm{CBPF}$.
} 


\section{Introduction}

Despite the great success of the standard model there are some phenomena, such as small mass for the neutrino, which can not be described by this model and ask for an extension. It is our aim in this work to propose a mass generation mechanism without the need of introducing a Higgs-type scalar field. From hidden directions of a higher-dimensional background: When we fill this background with massless Majorana modes and break it into two parts, such that one of the parts is the $(1+3)$-dimensional space-time, we naturally get mass and we obtain results which resemble those appearing in the context of the See-Saw Mechanism. In the present contribution, we extended this approach and the results obtained by Viollier et al some years ago [1], adopting a model with $D=(5+5)$ space-time dimensions as our higher-dimensional space-time. We consider the possibility of applying this approach as a mechanism for neutrino mass generation. In particular, our proposal fits into a series of works that review the potential use of models with extra time dimensions $[2,3,4,5]$.

\section{Building up the Space-Time: $D=(5+5)$ Dimensions}

Our background will be a space-time with five space and five time directions, that is $\mathrm{D}=(5+5)$, with a pseudo-Euclidean metric as given below. The connection between a $(5+5)$ and a $(2+2)$ space-time with string dynamics has been discussed by Ketov, Nishino and Gates in the work of [6]. For another motivation, we can also recall that quantum consistency of the superstring requires 10 space-time dimensions, but not necessarily in the usual $(1+9)$ signature. The signature is not completely arbitrary, however, since spacetime supersymmetry allows only $(1+9),(5+5)$ or $(9+1)$. Unfortunately, superstrings have as yet no answer to the question as of why our universe appears to be four-dimensional, neither why it appears to have signature $(1+3)$ [7].

As usual, $(+)$ is related to time directions and (-) to space directions. The metric is given by

$$
g_{\mu v}=\operatorname{diag}(1,-1,-1,-1,1,1,-1,1,-1,1) .
$$

The gamma-matrices, in this space-time, obey the following Clifford algebra

$$
\left\{\Gamma_{\mu}, \Gamma_{v}\right\}=2 g_{\mu v} .
$$

We can write down a possible set of $\left(2^{5} \times 2^{5}\right)$ gamma-matrices, in the Weyl representation [8], satisfying the Clifford algebra given in Eq. (2.1):

$$
\begin{aligned}
& \Gamma_{1}=i \sigma_{x} \otimes \sigma_{x} \otimes \sigma_{x} \otimes \sigma_{x} \otimes i \sigma_{y}, \\
& \Gamma_{2}=i \sigma_{x} \otimes \sigma_{x} \otimes \sigma_{x} \otimes \sigma_{x} \otimes \sigma_{x}, \\
& \Gamma_{3}=i \sigma_{x} \otimes \sigma_{x} \otimes \sigma_{x} \otimes \sigma_{x} \otimes \sigma_{z}, \\
& \Gamma_{4}=i \sigma_{x} \otimes \sigma_{x} \otimes \sigma_{x} \otimes \sigma_{z} \otimes \mathbb{I}_{2}, \\
& \Gamma_{5}=i \sigma_{x} \otimes \sigma_{x} \otimes \sigma_{x} \otimes i \sigma_{y} \otimes \mathbb{I}_{2}, \\
& \Gamma_{6}=i \sigma_{x} \otimes \sigma_{x} \otimes i \sigma_{y} \otimes \mathbb{I}_{2} \otimes \mathbb{I}_{2}, \\
& \Gamma_{7}=i \sigma_{x} \otimes \sigma_{x} \otimes \sigma_{z} \otimes \mathbb{I}_{2} \otimes \mathbb{I}_{2}, \\
& \Gamma_{8}=i \sigma_{x} \otimes i \sigma_{y} \otimes \mathbb{I}_{2} \otimes \mathbb{I}_{2} \otimes \mathbb{I}_{2}, \\
& \Gamma_{9}=i \sigma_{x} \otimes \sigma_{z} \otimes \mathbb{I}_{2} \otimes \mathbb{I}_{2} \otimes \mathbb{I}_{2}, \\
& \Gamma_{10}=-\sigma_{y} \otimes \mathbb{I}_{2} \otimes \mathbb{I}_{2} \otimes \mathbb{I}_{2} \otimes \mathbb{I}_{2}, \\
& \Gamma_{11}=\sigma_{z} \otimes \mathbb{I}_{2} \otimes \mathbb{I}_{2} \otimes \mathbb{I}_{2} \otimes \mathbb{I}_{2} .
\end{aligned}
$$


The 32-component Majorana spinors will be denoted by $\Psi$. The 16-component Majorana-Weyl spinors, that appear associated to one of the two off-diagonal block of the gamma-matrices, may be distributed in four (4-components) spinors referred to as $\chi_{N}, N$ taking the values $(1,2,3,4)$. Looking for five gammas-matrices associated to the Clifford algebra of the (1+3)-dimensional spacetime, we can find them as the last tensor product in each one of the fifth first equations of the set given by Eq. (2.2). This gammas-matrices are organized as $\left(\gamma_{\alpha} ; \gamma_{5}\right), \alpha$ sweeping the values $(1,2,3,4)$ and

$$
\begin{aligned}
& \gamma_{1}=i \sigma_{x} \otimes i \sigma_{y}, \\
& \gamma_{2}=i \sigma_{x} \otimes \sigma_{x}, \\
& \gamma_{3}=i \sigma_{x} \otimes \sigma_{z}, \\
& \gamma_{4}=i \sigma_{z} \otimes \mathbb{I}_{2}, \\
& \gamma_{5}=i \sigma_{y} \otimes i \mathbb{I}_{2} .
\end{aligned}
$$

\section{Filling up the Space-Time}

The massless Dirac equation of a (5+5)-dimensional space-time reads as follows:

$$
i \Gamma_{\mu} \partial^{\mu} \Psi=0, \mu=1, \ldots, 10 .
$$

In terms of the spinors $\chi_{N}$ and the 4-dimensional space-time matrices $\gamma_{\mu}$, we can write one of the off-diagonal blocks of Eq. (3.1) as

$$
i\left(\begin{array}{cc|cc}
\gamma_{\alpha} \partial^{\alpha}-i \mathbb{I}_{4} \partial^{6}+\gamma_{5} \partial^{5} & -i \mathbb{I}_{4} \partial^{7}-i \mathbb{I}_{4} \partial^{8} & 0 & -i \mathbb{I}_{4} \partial^{9}+i \mathbb{I}_{4} \partial^{10} \\
i \mathbb{I}_{4} \partial^{7}-i \mathbb{I}_{4} \partial^{8} & \gamma_{\alpha} \partial^{\alpha}+i \mathbb{I}_{4} \partial^{6}+\gamma_{5} \partial^{5} & -i \mathbb{I}_{4} \partial^{9}+i \mathbb{I}_{4} \partial^{10} & 0 \\
\hline 0 & i \mathbb{I}_{4} \partial^{9}+i \mathbb{I}_{4} \partial^{10} & \gamma_{\alpha} \partial^{\alpha}-i \mathbb{I}_{4} \partial^{6}+\gamma_{5} \partial^{5} & -i \mathbb{I}_{4} \partial^{7}+i \mathbb{I}_{4} \partial^{8} \\
i \mathbb{I}_{4} \partial^{9}+i \mathbb{I}_{4} \partial^{10} & 0 & i \mathbb{I}_{4} \partial^{7}+i \mathbb{I}_{4} \partial^{8} & \gamma_{\alpha} \partial^{\alpha}+i \mathbb{I}_{4} \partial^{6}+\gamma_{5} \partial^{5}
\end{array}\right)\left(\begin{array}{c}
\chi_{1} \\
\chi_{2} \\
\chi_{3} \\
\chi_{4}
\end{array}\right)=0
$$

Assuming a plane wave solution for the Majorana spinor in $\mathrm{D}=(5+5)$ dimensions,

$$
\Psi=u e^{-i P_{\mu} x^{\mu}},
$$

we have that the relativistic energy-momentum equation is $P_{\mu} P^{\mu}=0$. Constraining the invariant of mass $m$ to (1+3)-dimensional space-time, we have

$$
\left(P^{1}\right)^{2}-\left(P^{2}\right)^{2}-\left(P^{3}\right)^{2}-\left(P^{4}\right)^{2}=m^{2}
$$

and

$$
\left(P^{5}\right)^{2}+\left(P^{6}\right)^{2}-\left(P^{7}\right)^{2}+\left(P^{8}\right)^{2}-\left(P^{9}\right)^{2}+\left(P^{10}\right)^{2}=-m^{2} .
$$

From Eq. (3.2), we can read four coupled Dirac equations for $\mathrm{D}=(1+3)$ space-time,

$$
\left(\mathbb{I}_{4} \otimes i \gamma_{\alpha} \partial^{\alpha}+M\right) \chi_{N}=0 ; \quad \alpha=1, \ldots, 4 ; \quad N=1, \ldots, 4,
$$

where the mass matrix is given by $M=M^{\prime}+M^{\prime \prime}$, where 


$$
M^{\prime}=\left(\begin{array}{cc|cc}
\gamma_{5} P^{5} & 0 & 0 & 0 \\
0 & \gamma_{5} P^{5} & 0 & 0 \\
\hline 0 & 0 & \gamma_{5} P^{5} & 0 \\
0 & 0 & 0 & \gamma_{5} P^{5}
\end{array}\right)
$$

and

$$
M^{\prime \prime}=\left(\begin{array}{cc|cc}
-i \mathbb{I}_{4} P^{6} & -i \mathbb{I}_{4} P^{7}-i \mathbb{I}_{4} P^{8} & 0 & -i \mathbb{I}_{4} P^{9}+i \mathbb{I}_{4} P^{10} \\
i \mathbb{I}_{4} P^{7}-i \mathbb{I}_{4} P^{8} & i \mathbb{I}_{4} P^{6} & -i \mathbb{I}_{4} P^{9}+i \mathbb{I}_{4} P^{10} & 0 \\
\hline 0 & i \mathbb{I}_{4} P^{9}+i \mathbb{I}_{4} P^{10} & -i \mathbb{I}_{4} P^{6} & -i \mathbb{I}_{4} P^{7}+i \mathbb{I}_{4} P^{8} \\
i \mathbb{I}_{4} P^{9}+i \mathbb{I}_{4} \partial^{10} & 0 & i \mathbb{I}_{4} P^{7}+i \mathbb{I}_{4} P^{8} & i \mathbb{I}_{4} P^{6}
\end{array}\right)
$$

Let us emphasize the fact that the mass matrix $M$ has a diagonal pseudoscalar, $M^{\prime}$, and a nondiagonal scalar mass, $M^{\prime \prime}$.

We get the following eigenvalues for the mass matrix

$$
\begin{aligned}
& m_{1}=P^{5}+\sqrt{\left(P^{5}\right)^{2}+m^{2}}, \\
& m_{2}=P^{5}-\sqrt{\left(P^{5}\right)^{2}+m^{2}}, \\
& m_{3}=-P^{5}+\sqrt{\left(P^{5}\right)^{2}+m^{2}}, \\
& m_{4}=-P^{5}-\sqrt{\left(P^{5}\right)^{2}+m^{2}} .
\end{aligned}
$$

It is useful to illustrate our discussion presenting a Table 1 with the eigenvalues of the mass matrix, (3.6) - (3.9), and various choices for $P^{5}$.

\begin{tabular}{|c|c|c|c|}
\hline & $P^{5} \rightarrow 0$ & $P^{5} \rightarrow \infty$ & $P^{5} \gg m$ \\
\hline$m_{1}$ & $m$ & $\infty$ & $2 P^{5}$ \\
\hline$m_{2}$ & $-m$ & 0 & $-\frac{m^{2}}{2 P^{5}}$ \\
\hline$m_{3}$ & $m$ & 0 & $\frac{m^{2}}{2 P^{5}}$ \\
\hline$m_{4}$ & $-m$ & $-\infty$ & $-2 P^{5}$ \\
\hline
\end{tabular}

Table 1: Eigenvalues of the mass matrix with different choices for $P^{5}$

We notice that whenever $P^{5} \rightarrow 0$, we get degenerate massive Dirac equations. $P^{5} \rightarrow \infty$ generates masses of two types: one almost zero and a very massive one in $(1+3)$-dimensions.

Please notice that, in our Table, if $P^{5} \gg m$, we have

$$
\begin{aligned}
& m_{1} \approx 2 P^{5}, \\
& m_{3} \approx \frac{m^{2}}{2 P^{5}} .
\end{aligned}
$$

This sets up a mechanism similar to the usual See-Saw Mechanism for neutrino masses [9], so that this proposal could allow us to understand the generation of hierarchies for the fermion masses in (1+3)-dimensions.

Finally, in Figure 1, we present the eigenvalues of the matrix mass as a function of the momentum $P^{5}$. 


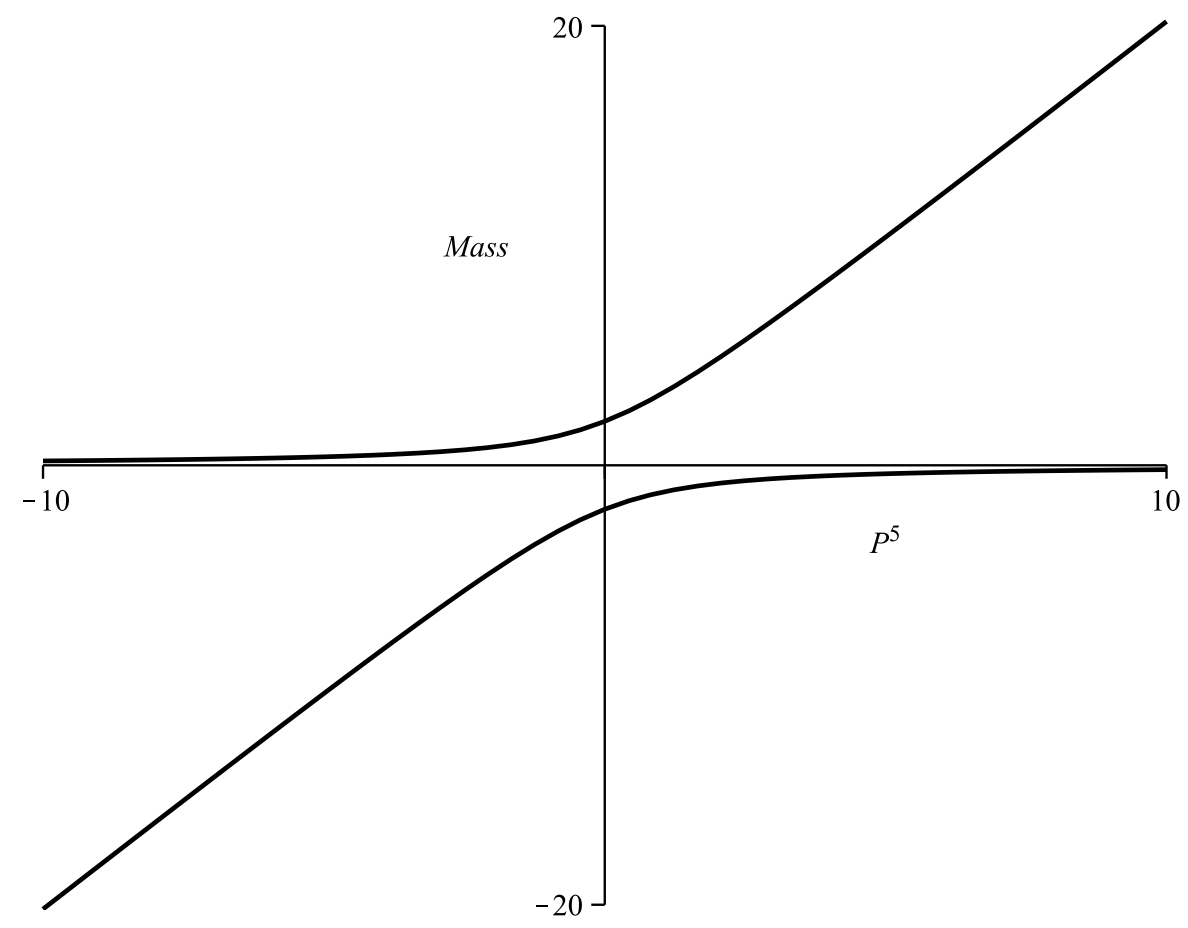

Figure 1: The eigenvalues of the mass matrix in $D=1+3$ dimensions for a fixed $m=4$.

\section{Conclusions}

We have shown that the Dirac equation, if formulated for non-interacting Majorana-Weyl spinors in $D=5+5$, may lead to an interpretation of massive Majorana spinors in $D=1+3$, after a suitable dimensional reduction. We confirm the process of dimensional by reducing extra dimensions as a mechanism to generate mass. The eigenvectors of the interaction or mass matrix are two particles of opposite chiralities and different masses moving in (1+3)-dimensions.

The eigenvalues of the mass matrix depend on the energy $P^{5}$ (associated the matrix of quiralidade $\gamma^{5}$ ) and the invariant mass $m$ of the four-dimensional spacetime. Let us analyze the most important results for the eigenvalues. First, when there is no interaction $\left(P^{5}=0\right)$, we have four particles with degenerate masses. Second, taking the limit $P^{5} \rightarrow \infty$, we obtain a massless along with heavy chiral particles. Finally, when $P^{5} \gg m$, we have two Majorana particles of masses $2 P^{5}$ and $\frac{m^{2}}{2 P^{5}}$, respectively. This result is in agreement with the See-Saw Mechanism for neutrinos masses. This mechanism of dimensional reduction for the generation of Majorana mass can be used to obtain the See-Saw masses for the fermions. Finally, we point out that this proposal allows us to understand the generation of hierarchies for the fermionic masses in (1+3)-dimensions. 


\section{Acknowledgments}

L. P. G. De Assis is grateful to FAPERJ for his post-doctoral fellowship and he would like to thanks the hospitality of CBPF.

M. Rojas thanks at CBPF for hospitality and acknowledges the support from FAPEMIG under the grant CEX 00311/08.

\section{References}

[1] R. D. Viollier, Amand Faessler and F. G. Scholtz, Mod. Phys. Lett. A 4 (1989) 2705.

[2] J. G. Foster and B. M. Müller, arXiv:1001.2485.

[3] Lanciani, P.: Found. Phys. 29 (1999) 251.

[4] Boyling, J.B., Cole, E. A. B. Int. J. Theor. Phys. 32 (1993) 801.

[5] Patty, C.E., Smalley, L.L. Phys. Rev. D 32 (1985) 891.

[6] Sergei. V. Ketov, Hitoshi Nishino and S. James Gates,Jr, Nucl. Phys. B 393 (1993) 149.

[7] M. J. Duff, ”Supermenbranes,” TASI Lecture Notes 1996, hep-th/9611203.

[8] H.L. Carrion, M. Rojas and F. Toppan, JHEP 0304 (2003) 040.

[9] Pavel Fileviez Perez, Tao Han, Guiyu Huang, Tong Li and Kai Wang, Phys. Rev. D 78 (2008) 015018. 